\title{
Diagnóstico prenatal: Labio leporino; catástrofe en la sala de partos
}

\author{
Alvaro Cano*; Andrés Sarmiento**
}

\begin{abstract}
RESUMEN: Se realiza una revisión de las hendiduras faciales desde la óptica obstétrica. Se hace énfasis en la importancia del diagnóstico sonográfico prenatal temprano como medio para optimizar el manejo neonatal y preparar a la familia.

PALABRAS CLAVES: Labio leporino, diagnóstico prenatal, cromosomopatías.

SUMMARY: A review of facial clefts from the obstetric point of view is practiced. The importance of early prenatal diagnosis is emphasized as a way to optimize neonatal care and preparation of the family.
\end{abstract}

KEY WORDS: Hare lip, chromosomophaties.

El desafortunado término de "labio leporino" ha sido utilizado ancestralmente para referirse al labio hendido o queilosquisis, estigmatizando a los pacientes afectados por esta alteración. A pesar que la queiloschisis es una malformación congénita relativamente frecuente, su diagnostico habitualmente se realiza tardíamente en la sala de partos. Precisamente es este escenario, tradicionalmente lleno de grandes expectativas el menos indicado para el diagnóstico de una alteración de tanta trascendencia estética y funcional, y que desafortunadamente implica un señalamiento social. Es frecuente que el obstetra y el pediatra no se encuentren debidamente preparados para informar idóneamente a los padres acerca del significado y de las repercusiones de esta malformación. Conscientes que el estado actual del diagnóstico prenatal y específicamente el ultrasonido de alta resolución permiten la adecuada valoración de la cara fetal, hemos querido en las siguientes líneas hacer una breve revisión desde la óptica obstétrica de la queiloschisis o "labio leporino".

\section{Reseña histórica}

El término "leporino" proviene del latín "lepus", adjetivo que hace referencia a la semejanza a una liebre. El término se remonta al siglo XVI cuando un médico francés valorando un paciente con labio hendido, acuño el término "leporino" refiriéndose a la similitud del labio afectado con la hendidura facial propia de la liebre y de otros roedores. Desafortunadamente en aquellos tiempos la liebre había sido relacionada con actos de superstición y brujería. Durante el oscurantismo se creía que las

\footnotetext{
* Residente de $4^{*}$ año, Escuela Colombiana de Medicina/Hospital Simón Bolivar.

Coordinador Académico Unidad de Medicina Materno Fetal, Escuela Colombiana de Medicina/Hospital Simón Bolívar. Director Científico OBGYN LTDA.
}

brujas podían tomar la forma de liebres. Esta nefasta circunstancia llevó a la creencia que si una liebre asustaba a una mujer embarazada, ésta daría a luz un recién nacido con el defecto como marca. Posteriormente, durante el siglo XVII, la histeria desatada por propagación de las prácticas de brujería terminó por asociar a la liebre con Satanás. Se llegó a considerar que una mujer quien daba a luz un producto con labio leporino, había mantenido relaciones con Satanás. El recién nacido o "semilla del mal" era considerado como una evidencia irrefutable de su relación pecaminosa con el mal. Entonces la madre era condenada a un trágico fin.

Ajenos a este oscuro origen y significado, hoy en día utilizamos el término "labio leporino" frecuentemente para describir la hendidura facial que puede involucrar labio y/o paladar (queiloschisis o palatoschisis). Sin embargo, el término se antoja estigmatizante para los padres y el recién nacido que frecuentemente perciben y reciben el rechazo social que aun evoca esta inadecuada y odiosa expresión.

\section{Incidencia}

El labio hendido con o sin paladar hendido y el paladar hendido aislado deben estudiarse como entidades aisladas. Difieren desde el punto de vista etiología, momento de aparición en el desarrollo embriológico y riesgos genéticos.

La queiloschisis es la malformación facial mas común y la segunda anomalía congénita mas frecuente después del pie Chapin. La incidencia de las anormalidades congénitas que comprometen el labio y el paladar varían de acuerdo con el área geográfica y al grupo étnico estudiado. En términos globales la incidencia no sindrómica de lesión labial con o sin compromiso del paladar ha sido estimada en 1:800-1000 nacidos. La lesión palatina aislada ocurre en 1:2500 nacidos. Se ha 
visto como en aproximadamente un $50 \%$ de afectados existe compromiso de labio y paladar. En el restante $50 \%$ están incluidos compromiso labial o palatino aislado en porcentajes similares.

El riesgo de recurrencia para estos defectos parece ser dependiente del tipo específico de daño. Por ejemplo, cuando se ha presentado un paciente con compromiso labial y palatino, el riesgo de recurrencia debe estimarse específicamente para este tipo de lesión. Luego de un primer producto afectado por lesión labial y palatina el riesgo de recurrencia es de aproximadamente un $4 \%$. Luego de dos hijos afectados, dicho riesgo se eleva al $9 \%$. Cuando uno de los padres y un primer producto son afectados, el riesgo de recurrencia puede ser del orden del 14-17\%. Por lo tanto, una historia familiar de hendidura labial y/o palatina debe poner en alerta al obstetra; la posibilidad de recurrencia está aumentada en 40-170 veces y debe ser una indicación absoluta para la cuidadosa valoración prenatal de la cara fetal en un siguiente embarazo.

\section{Epidemiología}

Aun cuando existen más de 180 síndromes relacionados con hendiduras de labio y paladar, estos solo representan un 5-10\% de casos. El restante corresponde a lesiones esporádicas de comportamiento epidemiológico muy variable. Las lesiones parecen ser mas frecuentes en grupos poblacionales como los Japoneses y algunas tribus indígenas de Alaska. La raza negra presenta una menor incidencia en relación a los caucásicos. El sexo masculino tiende a verse mas afectado y especialmente en casos mas severos (relación 2:1). El sexo femenino se ve mas afectado en casos de paladar hendido aislado (2:1). En un $75 \%$ de los casos existe compromiso labial (labio más paladar en un $50 \%$ o labio aislado en un $25 \%$ ).

\section{Etiología}

La etiología en casos aislados y no sindrómicos de labio y/o paladar hendido es incierta; parece ser una combinación multifactorial de elementos genéticos y ambientales.

Se ha propuesto que la aparición de labio hendido con o sin paladar (LH/P) y paladar hendido $(\mathrm{PH})$ aislado, pueden clasificarse en tres grupos genéticos:

1. Anomalía aislada, no-familiar.

2. Etiología sindrómica monogénica, cromosómica o ambiental.

3. Antecedente de afección familiar en primer, segundo o tercer grado de consanguinidad.

El $75-80 \%$ de casos de LH/P o PH pertenecen al primer grupo. Se han descrito 184 síndromes la mayoría raros en los cuales el LH/P y el PH hacen parte del criterio diagnóstico. En estos casos es importante establecer el síndrome específico pues la posibilidad de recurrencias puede llegar a ser del orden del $25-50 \%$. Un porcentaje importante de casos se ve asociado a trisomías 13 y 18 . Los factores ambientales pueden ser teratógenos (fenitoina, ácido valproico, alcohol, aminopterina o talidomida) o el síndrome de banda amniótica. En estos casos el riesgo de recurrencia es mínimo. En casos de herencia familiar han sido reportados patrones autosómico dominante (por ejemplo el Síndrome de Van der Woude) $\mathrm{y}$ recesivo ligado al $\mathrm{X}$.

\section{Embriología}

Entre las semanas 4 y séptima de gestación y a partir del quinto arco branquial aparecen varios pliegues de tejido mesenquimal que forman elevaciones ectodérmicas que rodean el estoma oral primario. Estas estructuras darán origen a cinco prominencias: una fronto-nasal, dos mandibulares y 2 maxilares. Estas estructuras inicialmente se verán separadas por hendiduras; posteriormente estas últimas se obliterarán con el desarrollo de las prominencias. Las dos maxilares crecen medialmente para fusionarse con las porciones intermedias de la prominencia nasal, para formar el segmento intermaxilar. A este pertenecen el labio superior, el componente maxilar superior (incisivos y alvéolos) y el paladar primario. El labio hendido resulta de la inadecuada fusión de las prominencias con persistencia de las hendiduras.

Embriológicamente el paladar se divide en primario o secundario. El primero comprende el triángulo anterior (que incluye alvéolos y los cuatro incisivos); mientras que el secundario comprende el resto del paladar duro. El paladar se origina de la fusión de tres procesos palatinos. Uno medial que depende de la prominencia nasal y dos laterales que dependen de los procesos maxilares. El proceso palatino además debe fusionarse con el septo nasal. Este paso aparentemente ocurre una semana mas tarde en el sexo femenino. La inadecuada fusión de estas estructuras deriva en la existencia de paladar hendido.

\section{Patología}

Las hendiduras faciales comprenden un amplio espectro de malformaciones que varían enormemente en su severidad. Las lesiones pueden variar desde una simple úvula bífida, pasando por indentaciones lineales del labio, hendiduras submucosas del paladar blando a grandes defectos mucosos y óseos. El defecto en el labio hendido se extiende desde el borde labial hasta la fosa nasal. En el labio y paladar hendido el defecto compromete el borde alveolar hasta el paladar duro, llegando a incluir el piso de las cavidades nasales o inclusive el de la órbita.

El LH se presenta unilateral en $80 \%$ de casos. En casos unilaterales el compromiso del paladar ocurre en un $70 \%$ de las veces; o hasta en un $85 \%$ si el compromiso es bilateral.

Alrededor de $7-13 \%$ de casos con LH aislado nacen con anomalías asociadas. Este porcentaje aumenta al 14 $\%$ en casos de LH/P y se eleva hasta un $35 \%$ en presencia de PH. Las malformaciones mas frecuentemente asociadas a LH/P y PH son:

* Cara: Arrihnia, hipo-hipertelorismo, proboscis.

*SNC: Holoprosencefalia, hidrocefalia, porencefalia, agenesia del cuerpo calloso.

* Cardíacas: defectos septales.

* Renales: hidronefrosis, riñón poliquístico y agenesia renal. 
* Esqueléticas: pie Chapin, agenesia de radio o de sacro, polidactilia.

* Abdominales: hernia diafragmática, mega vejiga.

* Otras: polihidramnios, hipospadia.

\section{Clasificación}

\section{Labio hendido}

a. Unilateral o bilateral.

b. Completo (si compromete labio mas arco alveolar) o incompleto.

\section{Paladar hendido:}

a. Unilateral o bilateral.

b. Completo (incluye paladar duro y arco alveolar) o incompleto (paladar secundario).

\section{Diagnóstico sonográfico}

El diagnóstico prenatal de las hendiduras faciales es muy reciente. El primer caso se reportó en 1981 en un feto de 28 semanas de gestación. Aun hoy en día existen pocos estudios con suficiente número de casos que permitan establecer con certidumbre la certeza diagnóstica del ultrasonido para el diagnostico del LH.

El examen sonográfico de la cara fetal puede practicarse a partir de las 14-16 semanas de gestación. La justificación de valorar sistemáticamente la cara fetal en embarazos de bajo riesgo no ha sido demostrada plenamente. Puede convertirse en un examen dispendioso que consume tiempo valioso, especialmente en el escenario congestionado de unidades de diagnóstico ecográfico. Requiere además de equipo actualizado, de un operador entrenado e incluso algunos autores argumentan lo poco productivo que resultaría examinar sistemáticamente la cara fetal en todo examen sonográfico.

Pilu refiere una sensibilidad del $78 \%$ con ultrasonido de detalle anatómico para la detección de anomalías faciales en una población de 233 pacientes con factores de alto riesgo para presentar malformaciones faciales. En $11 \%$ de fetos (con edades gestacionales entre las 18 y 40 semanas) no se logró obtener una adecuada visualización de la cara fetal en dos intentos. Las anomalías detectadas incluyeron polihidramnios, holoprosencefalia, proboscis, hipotelorismo, micrognatia y labio hendido con y $\sin$ paladar. Cuatro casos con compromiso aislado de paladar y tres de labio. Ningún feto con reporte ecográfico de cara normal presentó anomalías.

En otro estudio que incluyó 7100 pacientes de bajo y alto riesgo, la detección de anomalías cráneo-faciales en exámenes practicados entre las 20 y 39 semanas fue del $0.15 \%$. De los once casos detectados, 8 presentaban anomalías extra faciales que hicieron sospechar el diagnóstico. Siete fetos presentaron polihidramnios. Nuevamente, dos casos con labio hendido y 4 casos con paladar hendido no fueron detectados prenatalmente.

En un estudio más reciente, que incluyó 5407 pacientes sin factores de riesgo se practicó valoración sistemática de la cara fetal a la semana 22 y 34 de gestación. La certeza diagnóstica para anomalías faciales fue del $72 \%$. Cuando la anomalía facial se acompanaba de otras malformaciones extrafaciales la sensibilidad aumentó al 100\%.
Estos reportes sugieren que el diagnóstico prenatal de anomalías faciales es posible de realizar. Para el clínico y el sonologista la existencia de marcadores como ingesta de teratógenos, historia familiar de LH/P o PH y anomalías como polihidramnios u otras malformaciones extrafaciales en el embarazo actual, deben suponer un factor de riesgo que indica un examen pormenorizado de la cara fetal. En la casuística revisada el diagnóstico prenatal de la anomalía no cambió en forma importante el pronóstico neonatal. Finalmente la sensibilidad diagnóstica aun en población de alto riesgo no es la mejor, aun en manos experimentadas.

El examen sonográfico facial debe incluir:

1. Visualizar labio superior completo

2. Descartar protrusión de la lengua, apertura bucal persistente o regurgitación fetal.

3. Valoración de órbitas (hipo, hipertelorismo)

4. Valoración de región periorbitaria (descartar proboscis)

5. Valoración de las orejas (inserción baja, anomalías)

6. Valoración del perfil fetal.

En el diagnóstico sonográfico quizás el mejor acceso a la boca sean los planos frontal y coronal de la cara fetal. Las angulaciones del transductor en el sentido lateral, superior e inferior van a permitir una mejor visualización de mucosas, borde alveolar, mentón y nariz. La visualización en el plano frontal posterior del borde alveolar permitirá descartar la existencia de un paladar hendido. Este diagnóstico es algo mas dispendioso que el de labio hendido, el cual por lo general de existir tiende a ser mas obvio. La visualización de la cara fetal puede iniciarse por vía transvaginal a partir de las $10-11$ semanas. El perfil puede ser identificado a partir de la semana 14 , cuando también ya es posible identificar la integridad del labio superior. A partir de las 16 semanas es posible identificar el labio superior fetal mediante acceso transabdominal. La detección de la prominencia premaxilar se asocia en mas de un $90 \%$ con presencia de labio y paladar hendidos. Su diagnóstico se puede hacer tempranamente desde las 20 semanas y es un marcador muy útil en el diagnóstico de anomalías faciales. El labio hendido incompleto tiende a ser mas sutil para el diagnóstico; eventualmente solo se logra a finales del segundo trimestre. El paladar hendido aislado es aun mas difícil de detectar prenatalmente. Su diagnóstico depende del compromiso de estructuras del paladar duro. La lesión aislada del paladar blando puede pasar inadvertida al examen sonográfico. En caso de hendiduras mediales se debe practicar un examen exhaustivo de las estructuras del sistema nervioso central en busca de anomalías que pudieran sugerir una holoprosencefalia.

En presencia de un labio hendido con o sin paladar la coexistencia de anomalías cromosómicas es alta. En fetos con trisomía 13 la posibilidad de labio hendido alcanza un $60 \%$; mientras que en casos de trisomía 18 el riesgo puede ser de hasta un $20 \%$. Por lo tanto el diagnóstico ecográfico de un labio hendido es indicación absoluta de estudio cromosómico y estudio sonográfico de detalle en busca de otras anomalías. El síndrome de banda amniótica como causa de anomalías faciales usualmente comparte otras alteraciones dismórficas. 


\section{Importancia del diagnóstico prenatal}

El examen sistemático de la cara y boca fetal no está incluido en los criterios de la AIUM (American Institute of Ultrasound in Medicine) para el examen ecográfico obstétrico de rutina. Posiblemente lo relativamente infrecuentes que pueden ser las anomalías faciales, lo dispendioso que puede ser el examen facial fetal y el limitado entrenamiento de los operadores han hecho que la visualización facial no haya sido considerado una prioridad en el diagnóstico sonográfico.

A pesar de esto los continuos avances en el mejoramiento de las imágenes, el cada vez mejor entrenamiento de los operadores y el afán de obtener mayor información del examen anatómico fetal, han hecho que la tendencia actual sea incluir una valoración de la cara y boca fetal en todo examen sonográfico. En manos experimentadas y con un buen equipo esta evaluación solo tomara unos segundos, no aumentará costos y brindará estas potenciales ventajas:

1. En caso de detectarse una anomalía tipo labio hendido, preparar a los padres para un defecto estético importante pero que la mayoría de las veces tiene una solución quirúrgica definitiva. El parto se convierte en una situación donde se corrobora un diagnóstico y es el primer paso para iniciar el tratamiento definitivo.

2. Permite preparar a los padres para aceptar un hijo malformado y modificar la actitud usual inicial de rechazo, encubrimiento o de evasión.

3. Prepara el equipo quirúrgico y médico para la atención integral del neonato (cirujano plástico, psicólogo, neonatólogo, fonoaudiólogo, odontólogo, obstetra y genetista).

4. Las anomalías faciales son útiles marcadores para cromosomopatías u otras anomalías estructurales fetales. Pueden ser el primer hallazgo para diagnosticar un sindrome.

5. Algunas malformaciones como la macroglosia, micrognatia o el labio y paladar hendidos, son entidades que potencialmente pueden provocar problemas respiratorios severos durante la reanimación postparto.

Su diagnóstico prenatal permite la oportuna intervención en un parto programado del neonatólogo.

\section{Desventajas :}

1. Puede aumentar la ansiedad materna. Anticipa un diagnóstico para el cual poco o nada hay que ofrecer prenatalmente hoy en día.

2. Al incurrir en técnicas invasivas de diagnóstico cromosómico presume riesgos para el embarazo.

3. Eleva costos de tiempo y gastos de diagnóstico prenatal.

4. En casos excepcionales el diagnóstico prenatal mejora el pronóstico neonatal.

\section{Tratamiento actual}

El manejo ideal de el LH/P y $\mathrm{PH}$ requiere del concurso de un grupo de trabajo donde deben estar el obstetra, el neonatólogo, el cirujano, el ortodoncista, genetista, fonoaudiólogo, nutricionista y psicólogo. Este manejo obviamente debe envolver no solo el neonato sino a sus padres.
Los pormenores del manejo quirúrgico de estos pacientes escapa a los objetivos de esta revisión. El momento de la corrección quirúrgica depende de muchos factores y de la experiencia del cirujano plástico u otorrinolaringólogo encargado del caso. Lo usualmente aceptado es programar la corrección para las 10 semanas de vida o cuando las condiciones prequirúrgicas del paciente sean óptimas. Se han referido cierres primarios temporales "de adhesión" a los 10 días de vida. En casos de compromiso del paladar este se corrige a los 3-6 meses. Cuando existe compromiso del maxilar superior, este tratamiento puede postergarse hasta los 8 años de vida. Inclusive la corrección definitiva de defectos nasales puede demorarse hasta los 16-18 años.

Recientemente en el Hospital Simón Bolívar se ha propuesto la queiloplastia temprana en el recién nacido como una alternativa al manejo de pacientes con labio hendido. Durante el ultimo año se practicaron 25 correcciones de $\mathrm{LH}$ a recién nacidos a término y con peso $>$ a 2500 gramos, mediante técnicas de Millard y TennisonRandal. Se han obtenido buenos resultados con un inicio temprano (3-6 horas) del postoperatorio de la vía oral, mejoría del proceso respiratorio, mayor ganancia de peso a corto plazo, disminución en el rechazo de los padres y aumento del afecto materno.

\section{Conclusiones}

* El término "labio leporino" preferiblemente debe evitarse por su significado histórico y connotación social estigmatizante.

* Las hendiduras faciales (LH/P y PH) son anomalías relativamente frecuentes y de alta trascendencia estética y funcional. Son alteraciones teóricamente detectables mediante ultrasonido desde tan temprano como las 14 a 16 semanas. El diagnóstico prenatal hoy en día posee una certeza diagnóstica cercana al $75 \%$

* La detección de LH/P y/o PH es una indicación absoluta de diagnóstico citogenético y ultrasonido de detalle anatómico, por su alta asociación a cromosomopatías y alteraciones estructurales asociadas.

* La valoración facial fetal está absolutamente indicada en caso de dismorfismo fetal (cualquier anomalía anatómica pero especialmente polihidramnios y alteraciones del sistema nervioso central), antecedente de teratogenicidad o historia familiar positiva. Sin embargo en las series reportadas a la fecha, el diagnóstico prenatal no aportó beneficios neonatales significativos.

* La conveniencia de incluir la cara fetal en el examen obstétrico rutinario en población de bajo riesgo depende del criterio medico; pero indudablemente está asociado a la experiencia y habilidad del operador para obtener una visión óptima sin consumir mayor tiempo. La AUIM no incluye la cara fetal en el examen obstétrico de rutina.

* A medida que la sonografía prenatal evoluciona, el operador debe estar preparado para hacer diagnóstico de anomalías que hasta hace poco tiempo se consideraban difícilmente evidenciables. Si bien el diagnóstico prenatal de un labio hendido puede desencadenar ansiedad materna, esta misma puede causarse en madres cuyo sonologista no pueda decartarles la remota posibilidad de una anomalía tan indeseable como la hendidura facial. 
Fotografía 1

VISTA FRONTAL LABIO HENDIDO EN EMBARAZO DE 3ER TRIMESTRE.

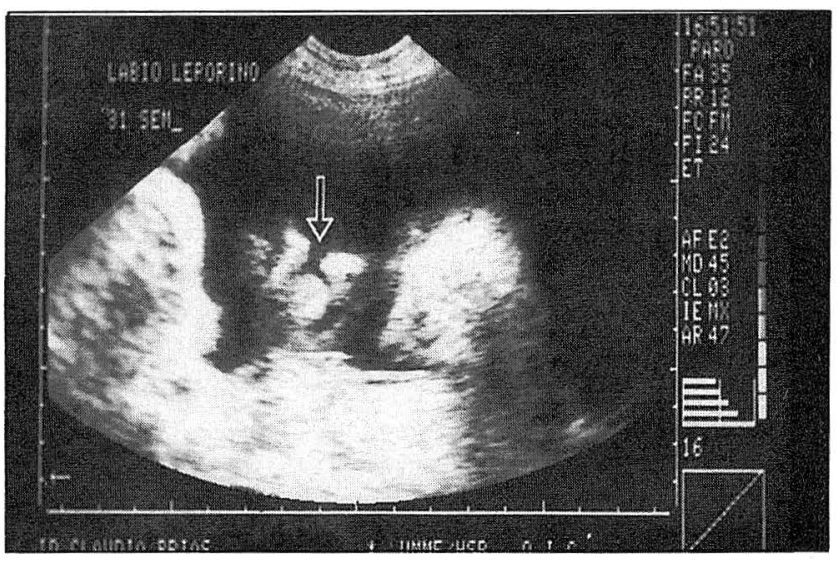

Fotografia 2

VISTA DE PERFIL DE LA CARA FETAL, PROMINENCIA NASAL Y BOCA, POR LITRASONIDO DE 2O. TRIMESTRE.

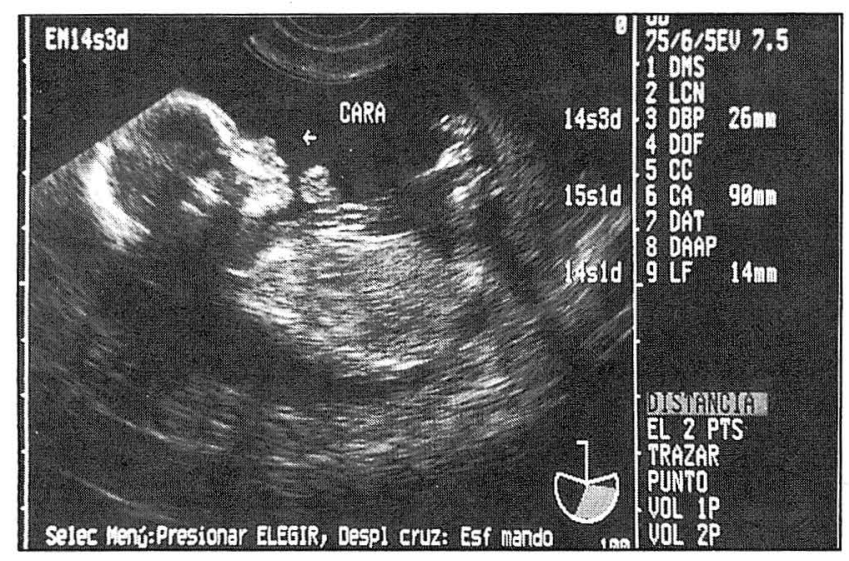

Fotografía 3.

VISTA EX CORTE CORONAL MODIFICADO DE LA PROMINENCIA NASAL, COANAS, LABIO SUPERIOR E INFERIOR Y MENTÓN POR ULTRASONIDO DE 3ER TRIMESTRE.

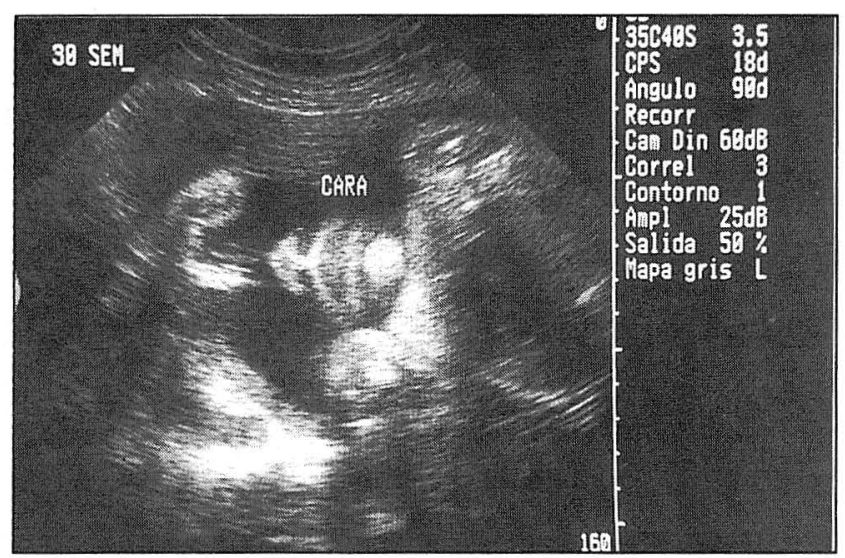

Fotografía 4.

VISTA DE CARA QUE PERMITE EVIDENCIAR INTEGRIDAD ANATÓMICA DE LOS TEJIDOS BLANDOS DE ILA BOCA FETAI.

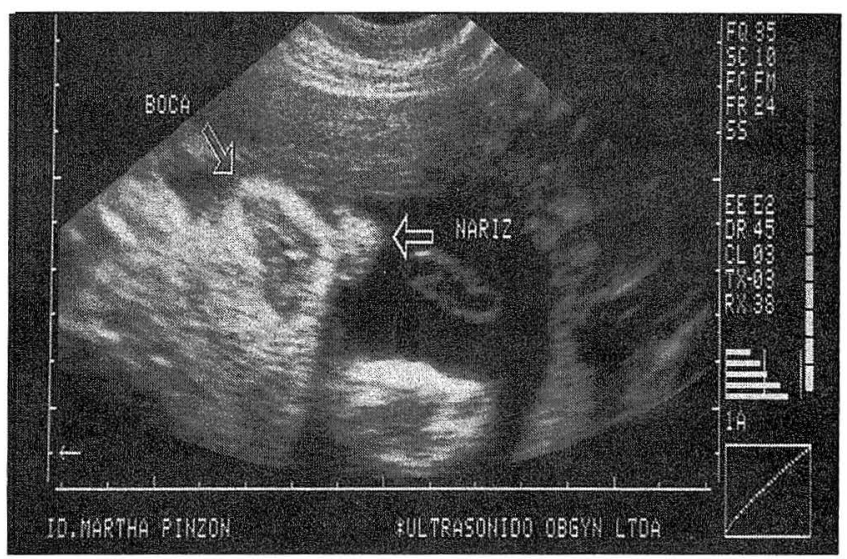

Fotografía 5

VISTA EN CORTE CORONAL MODIFICADO DE HENDIDURA LABIAL EN ULTRASONIDO DE 20 TRIMESTRE.

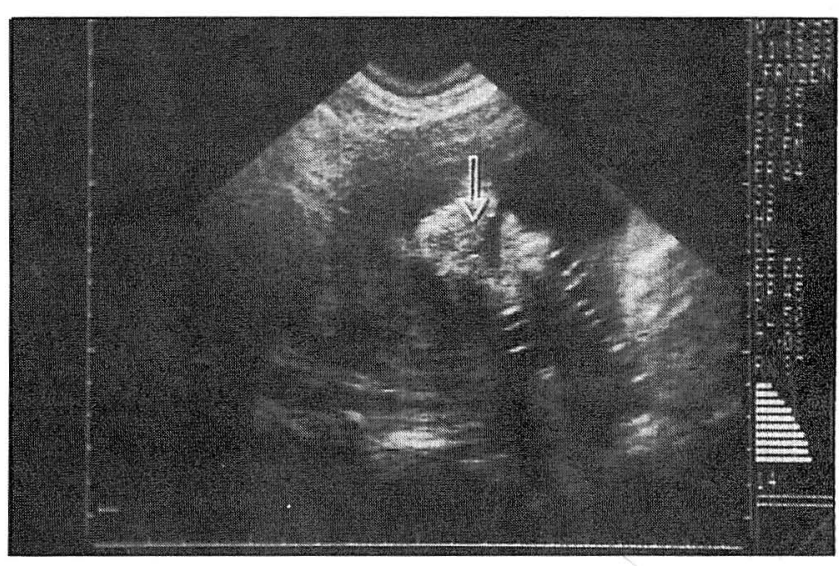

Fotografía 6.

VISTA CORONAI, FRONTAL DE LA CARA FETAI. QUE EVIDENCIA HENDIDURA LABIAL BILATERAI.

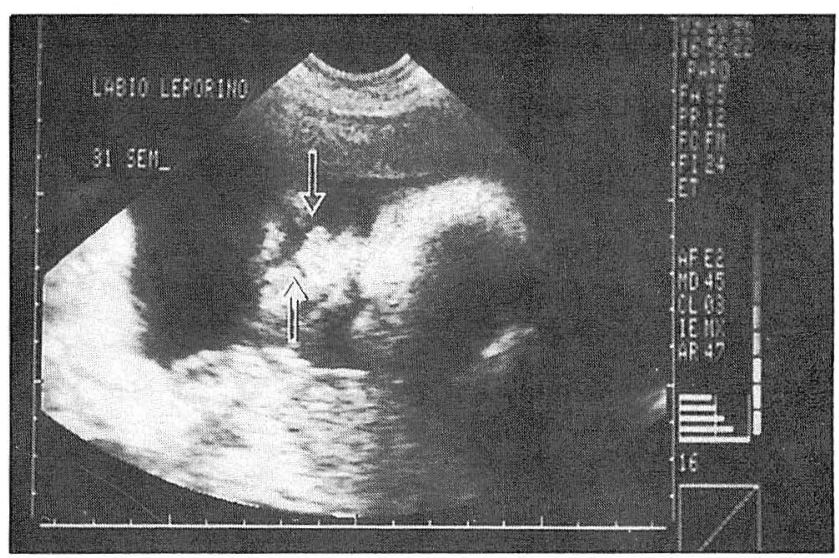




\section{BIBLIOGRAFIA}

1. Young Iam, Incidence of genetics of congenital malformations in "Prenatal Diagnosis and Screening - Congenital Malformations", Brack - Rodeck, Livingstone Ch De, 1992; 171-183.

2. Romero R., The Face in "Prenatal Diagnosis of Congenital Anomalies", Appleton \& Lange, 1988; 81-113.

3. Buyse Mary Louise, "Birth Deffects Encyclopedia - Cleft Lip and/or Cleft Palate", Blackwell De, 3rd Edition, 1994; 515-520.

4. Robinson A, Lindes E., "Clinical Genetics Handbook" 3rd Edition, Blackwell De, 1993

5. Guerra de la Espriella Miguel "Queilopalatosquisis; corrección temprana en el Hospital Simón Bolívar", Descripción de casos. (Sin Publicar).

6. WWW. Widwsmiles, "Hare Lip: the dark history of an infortunate word", Septiembre de 1997.

7. Fogh-Andersen P, M.D., "Epidemiology and Etiology of Clefts", Birth Defects: Original Articles Series, 1971; 7(7).

8. Smith F. L., Calhoun K.., "Cleft Lip and Palate", Department of Otolaryngology, UTMB, Grand Rounds, March, 1991.
9. Benaceraff B. "Ultrasonography in Obstetrics and Gynecology: Chapter 12: Ultrasound Evaluation of the Fetal Face", W.B. Saunders Company, \#rd Edition, 1994.

10. Nyberg A. D., et al. "Premaxillary Protrusion: a Sonographic Clue to Bilateral Cleft Lip and Palate", J Ultrasound Medicine, 1993; 12: 331335 .

11. Mahony B., Nyberg D., "Chapter 78: The Fetal Face, Ultrasoun in Obstetrics. F. Chevernak. 1993.

12. Benacerraf B., Mulliken JB. "Fetal Clef Lip and Palate: Sonographic Diagnosis and Postnatal Outcome", Plastic and Reconstructive Surgery, 1993; 92(6).

13. Hajner E. et al. "Prenatal Diagnosis of Facial Malformations", Prenatal Diagnosis, 1997; 17: 51-58.

14. Christ JE, Meininger MG, "Ultrasound Diagnosis of Cleft Lip and Cleft Palate Before Birth", Plastic and Reconstructive Surgery, 1981; 68(6).

15. Aucher Bruce, "Timing is Everithing", http:/// www.widesmiles.org, 1996.

16. Hovey ML. "Unilateral Cleft Lip Repair" http:///www.widesmiles.com

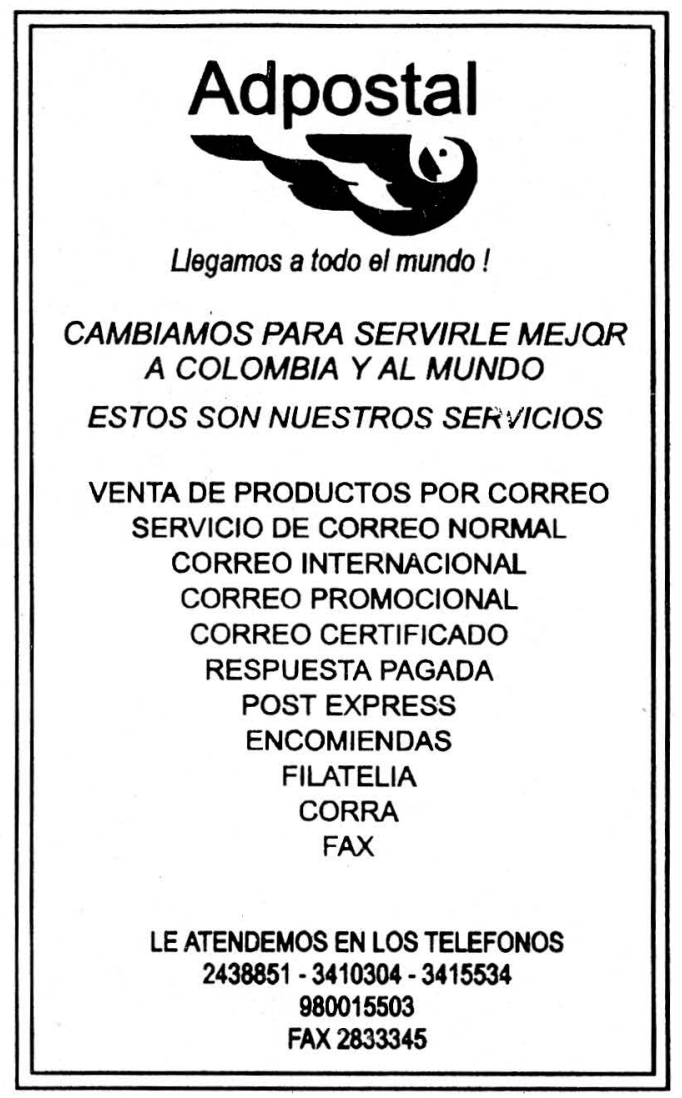

